\title{
Flexible Denture: A Hope for Partial Edentulous Patient-A Case Report
}

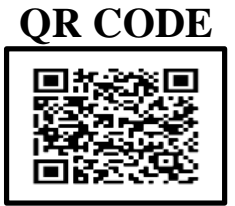

\section{GITANJALI MAGO*1, PUNEET SHARMA}

The various treatment options for the aesthetic and functional rehabilitation of partially edentulous patients are conventional fixed partial dentures, implant supported fixed partial dentures and removable partial dentures. In some cases, removable partial dentures may be the only choice which is available other than implants and fixed partial denture. Removable cast partial dentures are used as definitive removable prostheses when indicated, but location of clasps is not aesthetically pleasant. So, when patient is concerned about aesthetics, flexible partial dentures which is aesthetically superior may be considered. But for the success of flexible removable partial denture, proper diagnosis, treatment planning, insertion, wearing and maintenance of this prosthesis is very important.

KEYWORDS: Flexible Denture, Valplast, Flexiplast, Nylon, Acrylic Clasps

\section{INTRODUCTION}

The introduction of acrylic polymers and chrome cobalt alloys type of removable partial dentures have gained popularity among dentists. Decade ago, removable partial denture were chosen by the patients for their low cost and good physiology. Modern era has led to the evolvement of the removable partial denture from traditional hard fitting denture bases to soft dentures by using nylon based thermoplastic material. So they have become an excellent alternative to traditional removable partial dentures which have many problems like loss of chewing efficiency, hard denture bases and less retention. These flexible dentures not only provide good function and esthetics but are also comfortable and strong. The flexible nylon resin has gaining popularity because of its simplified design and a built- in stress breaker which provide better stress distribution and a superior function. So flexible denture has become a good option not only for the dentists but also for the patients. ${ }^{1-3}$

\section{CASE REPORT}

A 51-year female patient reported to the Department of Prosthodontics with a chief complaint of bad aesthetics due to the front missing teeth (Figure 1). The patient presented with multiple missing anterior teeth (Kennedy's class IV) (Figure 2). She was very much apprehensive about the aesthetics and did not want any rigid or metal prosthesis in her mouth.

\section{PROCEDURE}

- Firstly, alginate impressions were made and diagnostic casts were prepared (Figure 3).

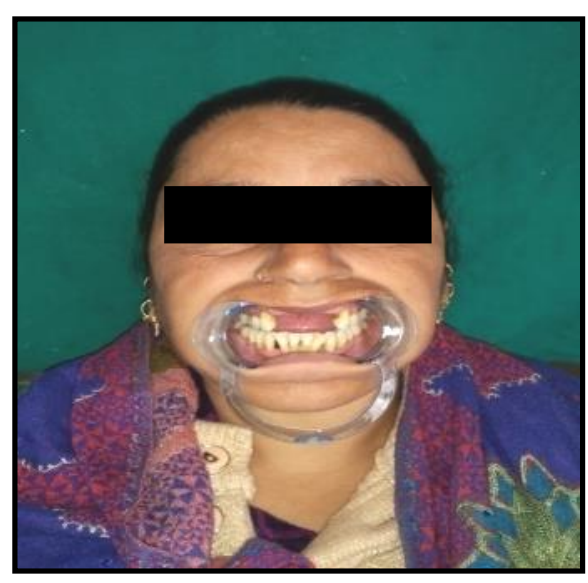

Figure 1. Preoperative view

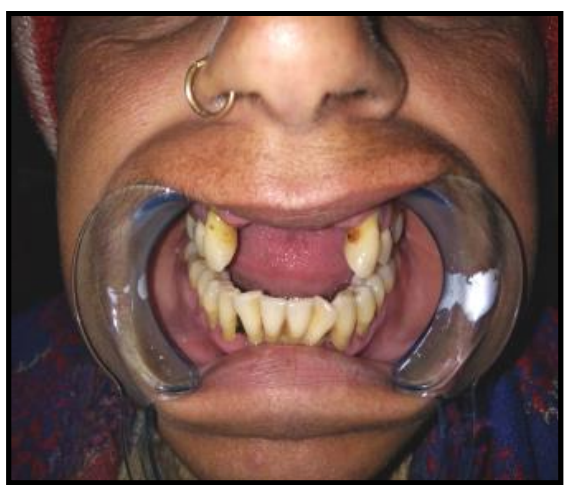

Figure 2. Intraoral view 


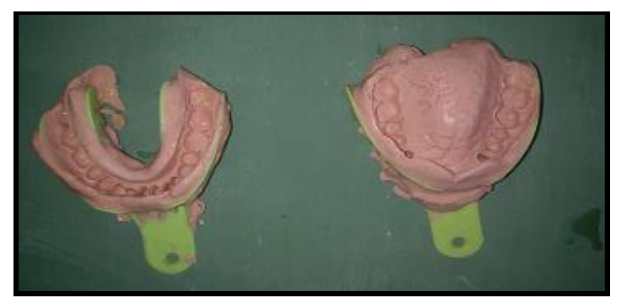

Figure 3. Impression of the arches

- Cast were mounted on surveyor and were analyzed for presence of any undercuts.

- After that using centric relation record and face bow transfer to evaluate inter-arch space the diagnostic casts were articulated on the articulator.

- Final impressions were made for both the arches using polyvinyl siloxane light body material and final casts were made with Type III dental stone.

- Maxillomandibular relationships were recorded with check bite method

- Then the definitive casts were mounted on the articulator.

- Shade selection was done and artificial acrylic resin teeth were arranged according to that.

- Denture try in was done in the patient's mouth and after the full satisfaction of patient denture was processed in the injection system.

- Then the final denture was finished, polished and inserted. Occlusion was evaluated and adjusted if needed (Figure $4 \&$ 5).

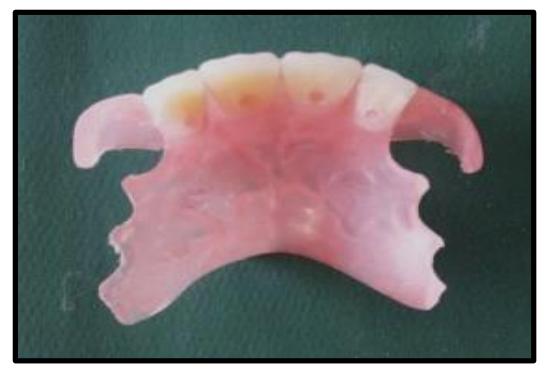

Figure 4. Palatal view of the flexible prosthesis

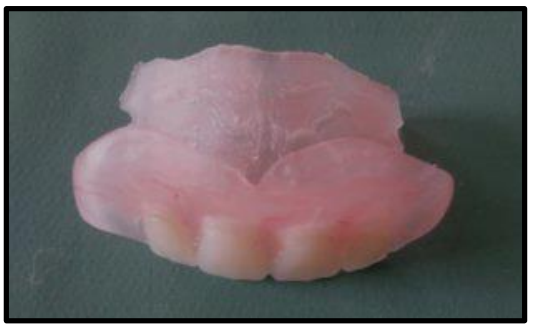

Figure 5. Labial view of the flexible prosthesis
- Postoperative instructions were given to the patient on how to insert the prostheses and with the need to maintain oral hygiene (Figure 6,7 \& 8).

\section{DISCUSSION}

Removable partial denture is commonly used for treating the patients who cannot be opted for conventional fixed partial dentures and implant supported prosthesis. Since decades the removable cast partial denture has been used for rehabilitation of partially edentulous patients and its fabrication involves the use of metal alloy, acrylic resin and/or thermoplastic resins. In the first kind, i.e. a metal alloy consists of a metal base with acrylic teeth attached to it and metal retentive clasp that aids to hold the cast partial denture in position. But these metal clasps give metallic appearance which is not acceptable by many patients, especially those concerned about their aesthetics. In few cases where the maxillary posterior teeth are missing and only the anterior teeth are present, the placement of metallic clasps on canines may not be acceptable to patients due to its unpleasing design. ${ }^{4}$

The second type of removable partial denture as discussed above are all acrylic resin prosthesis, also known as temporary or interim removable partial denture or a "FLIPPER". It functions as a space maintainer and is commonly used to restore the function of the tooth during the treatment until the definitive prosthesis is fabricated in the laboratory.

Flexible denture material is available in the form of granules packed in cartridges of varying sizes and first introduced by the name of valplast and flexiplast since 1956. They are superpolyamides belonging to the family of nylon, a resin derived from dicarboxylic acid, diamine, amino acid and lactams. 5 The Injectionmolding technique is used for fabrication of flexible denture base prosthesis. ${ }^{8}$ A prosthesis fabricated from these materials has many advantages from the others as it requires minimum or no mouth preparation, good retention, comfortable for patient as it is thin and lightweight, resistant to fractures and is aesthetically pleasant because it is translucent and pink shade which matches with the natural tissues. Acrylic resin teeth do not tend to bond chemically with a flexible denture base resin and are retained by making $T$ shaped holes into which the resin flows to retain teeth mechanically. ${ }^{7}$ 


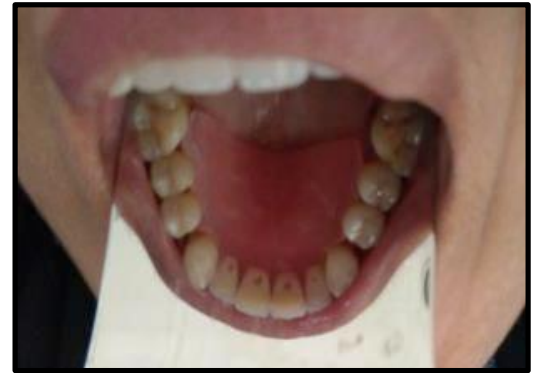

Figure 6. Intraoral palatal view of the flexible prosthesis in the patient's mouth

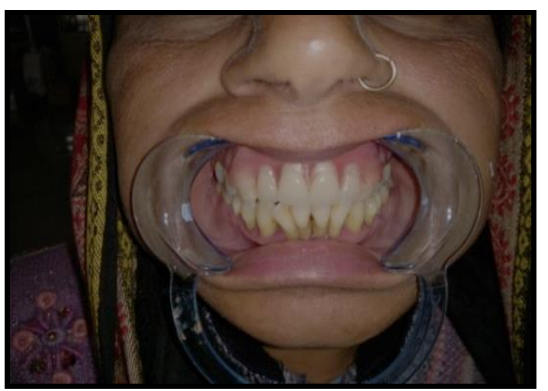

Figure 7. Intraoral frontal view of the flexible prosthesis in the patient's mouth

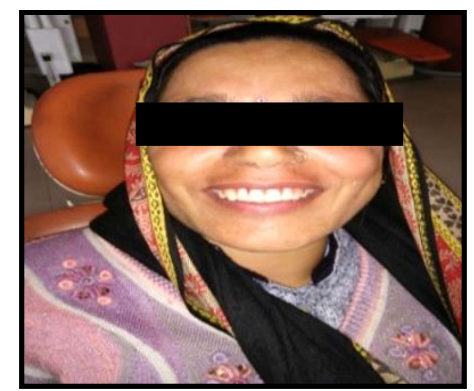

Figure 8. Postoperative view of the flexible prosthesis in patient's mouth
It hence, has been proven that flexible partial denture has several advantages over the other two types of partial dentures. Instead of using metal clasps, a flexible partial denture has a thin finger like extension extended into undercuts which act like a clasp. It is useful in patients having gingival recession as they appear elongated and for patients allergic to acrylic. ${ }^{2}$ However, fabrication of flexible partial and complete dentures is contraindicated in cases with insufficient inter-arch space i.e., less than $4 \mathrm{~mm}$ space, with prominent residual ridges where there is less space for labial placement of teeth because $\mathrm{T}$-shape holes are necessary for mechanical retention of teeth to denture base, and with flat- flabby ridges with poor soft tissue support which require more rigid prosthesis. ${ }^{3}$

As every invention has a few drawbacks, the flexible prosthesis is difficult to repair, reline, rebase and is prone to staining from tea and/or coffee if it is not polished properly and cleaned by the patient on a regular basis. In order to maintain the aesthetics and cleanliness of the prosthesis, the patient should be advised to practice good oral hygiene practices and clean his/her prosthesis regularly after every meal and it should be removed during brushing of natural teeth to avoid scratching of the prosthesis.

\section{CONCLUSION}

Flexible partial dentures are a good option for the replacement of missing teeth especially when patient is concerned about aesthetics. It should be borne in mind that proper care of the prosthesis is required in order to reduce staining of the prosthesis, as it affects the aesthetics of the prosthesis as time passes by. The fabrication depends on the clinician's skill in selection of the type of the prosthesis required for the patient. Flexible dentures while previously were selected by only a handful of patients and the clinician has gained its ground to become an effective and often demanded treatment option.

\section{REFERRENCES}

1. Thakral G, Aeran H, Yadav B, Thakral R. Flexible Partial Dentures - A hope for the Challenged Mouth. People's Journal of Scientific Research, 2012;5(2):55-9. 2. Singh K, Aeran H, Kumar N, Gupta N. Flexible Thermoplastic Denture Base Materials for Aesthetical Removable Partial Denture Framework. Journal of Clinical and Diagnostic Research, 2013; 7(10): 2372-3.

3. Jain AR. Flexible Denture for Partially Edentulous Arches - Case Reports. International Journal of Recent Advances in Multidisciplinary Research 2015; 2(1):1826.

4. Naylor WP, Manor RC. Fabrication of a flexible prosthesis for the edentulous scleroderma patient with microstomia. J. Prosthet Dent. 1983;50(4):536-8.

5. Yunus N, Rashid AA, Azmi LL, Abu Hassan MI. Some flexural. Properties of a nylon denture base polymer. J Oral Rehabil. 2005; 32:65-71.

6. Lowe LG. Flexible denture flanges for patients exhibiting undercut tuberosities and reduced width of the buccal vestibule: a clinical report. J. Prosthet Dent., 2004; 92(2):128-131.

7. Phoenix RD, Mansueto MA, Ackerman NA, Jones RE: Evaluation of mechanical and thermal properties of commonly used denture base resins. Journal of Prosthodontic, 2004; 13(1):17-27.

8. Parvizi A, Lindquist T, Schneider R. Comparison of the Dimensional Accuracy of Injection-Molded 
Denture Base Materials to that of Conventional Pressure-Pack Acrylic Resin. Journal of Prosthodontics 2004; 13(2):83-9.

9. Kutsch VK, Whitehouse J, Schermerhorn K, Bowers R. The evolution and advancement of dental thermoplastic. Dental Town Magazine. 2003.
10. Antonelli JR, Hottel TL. The "flexible augmented flange technique" for fabricating complete denture record bases. Quintessence Int. 2001;32(5):361-4.
Cite this article as:

Mago G, Sharma P. Flexible Denture- A Hope for Partial Edentulous Patient: A Case Report. Int Healthc Res J. 2019;3(8):267-270. https://doi.org/10.26440/IHRJ/0308.11302

\section{AUTHOR AFFILIATIONS:}

1. PG student, Department of Prosthodontics \& Implantology, D.J. College of Dental Sciences \& Research, Modinagar

(Corresponding Author: Dr. Gitanjali Mago)

e-mail of corresponding author: dr.gitanjalimago123[at]gmail[dot]com 